\title{
ANÁLISE DE ANTENAS DE ABERTURA ALIMENTADAS POR LINHAS DE FITA COM SUBSTRATOS UNIAXIAIS
}

\author{
A. V. Proaño De la Torre, J. C. da S. Lacava e Lucio Cividanes
}

\begin{abstract}
Resumo - Descreve-se neste trabalho um modelo dinâmico para a análise de antenas de abertura alimentadas por linhas de fita com substratos anisotrópicos uniaxiais, no caso em que o eixo óptico está orientado segundo a normal ao plano das interfaces. Utilizando o formalismo de Fourier, que encaminha o problema para o domínio espectral, são obtidas expressões simples e fechadas para as amplitudes dos campos transformados, nas diversas camadas que compõem a antena. São estabelecidas as equações integrais que possibilitam o cálculo. via método dos momentos, da densidade superficial de corrente magnética localizada no plano da abertura e da densidade superficial de corrente elétrica distribuida sobre a fita que a alimenta. A impedância característica, a constante de propagaçâo e o coeficiente de reflexão da referida linha de fita também são analisados. Efeitos da anisotropia uniaxial sobre os parâmetros acima citados são apresentados e discutidos. Além desses, são particularmente interessantes os ocasionados por essa anisotropia sobre a impedância de entrada de uma fenda retangular. Para estruturas com substratos isotrópicos, os resultados obtidos estão em boa concordância com os calculados por programas disponiveis comercialmente.
\end{abstract}

Palavras Chaves: Antenas de abertura, linhas de fita, anisotropia uniaxial, método dos momentos.

\begin{abstract}
This work presents a full-wave analysis method for printed apertures in the ground plane of striplines with uniaxial substrates, assuming the optical axis normal to the planar interfaces. Working in the Fourier domain, closed form expressions for the transformed electromagnetic fields are derived. Coupled integral equations are formulated to calculate the magnetic current density across the aperture and the electric current density on the stripline. The solutions for theses equations are obtained using the method of moments in the spectral domain. Results for characteristic impedance and propagation constant of the stripline are discussed. Effects of the anisotropic ratio $\left(\varepsilon_{\Sigma} / \varepsilon_{x}\right)$ on the normalized input impedance of a rectangular slot antenna are analyzed. As a result of our calculation it is clear that increasing this anisotropic ratio the antenna input impedance is affected by a non-negligible quantity, while the resonant frequency decreases. For isotropic structures, the numerical results are in good agreement with those computed by commercial $C A D$ programs.
\end{abstract}

A. V. Proaño De la Torre é da Escuela Politécnica del Ejército, Ecuador. J. C. da S. Lacava é do Instituto Tecnolóico da Aeronáutica. Lucio Cividanes do Instituto Nacional de Pesquisas Espaciais.

E-mails: anatorre@li.fie-espe.edu.ec, lacava@ele.ita.br, lucio@dea.inpe.br. Editor de Área responsável: Denise Consonni. Artigo recebido em 02/Mar/2001, revisado em: 24/Out/2001, aceito em 05/Jun/2002.
Keywords: Aperture antennas, striplines, uniaxial anisotropy, method of moments.

\section{INTRODUÇÃO}

Antenas de microfita, por apresentarem baixo perfil aerodinâmico, massa e volume reduzidos, além de se adaptarem com facilidade a superficies curvas, têm merecido atenção especial da engenharia aeroespacial brasileira. Microfitas retangulares têm sido utilizadas na confecçào de antenas para GPS (Global Positioning System), de redes cilíndricas para canais de telemetria de foguetes (foguetes Sonda $I V$ e $V L S)$, antenas para telemetria em ensaios em vôo (aeronave $A T-26$ Xavante) e antenas circularmente polarizadas para pequenos satélites científicos [1-5]. Entretanto, esse tipo de antena apresenta algumas desvantagens operacionais tais como baixa capacidade de potência e elevado fator de qualidade. Essa última implica em uma estreita faixa de operação, tipicamente da ordem de $1 \%$ [6], o que limita a sua aplicação. Em adição a este fato, sabe-se que muitos dos substratos utilizados na confecção dessas antenas apresentam um certo grau de anisotropia [7]. Como consequiência, seus parâmetros elétricos são bastante afetados, em especial a sua freqüência de ressonância [8-11].

Se, por um lado, a antena de microfita possui uma operação tipicamente faixa estreita, por outro lado, uma abertura eletromagnética localizada no plano de terra de uma microfita pode apresentar um meihor desempenho. Além da faixa de operação, podem ser citadas como vantagens desse tipo de antena a baixa interação via onda de superfície, melhor isolação e irradiação desprezivel do sistema de alimentação [12]. Uma evolução dessa estrutura é a antena de microfita alimentada por uma abertura [13]. Entretanto, tais estruturas podem produzir diagramas traseiros de irradiação, que são incompatíveis com aplicações onde a antena necessita estar moldada ou simplesmente apoiada sobre superficies condutoras, como por exemplo em aeronaves, foguetes e satélites. Com o objetivo de eliminar esta irradiação traseira não desejada, uma possibilidade é a substituição da microfita de alimentação por uma linha de fita. Uma aplicação para este tipo de estrutura é a confecção de antenas ativas com feixes múltiplos, de grande interesse no setor aeroespacial.

Neste trabalho é apresentado um modelo dinâmico para a análise de antenas de abertura alimentadas por linhas de fita com substratos anisotrópicos uniaxiais. Os campos eletromagnéticos, nas diversas camadas, são determinados empregando-se a transformada dupla de Fourier que encaminha a solução do problema para o domínio espectral. Em conseqüência, são obtidas as funções de Green espectrais, o que possibilita uma simplificação considerável da aplicação do método dos momentos, uma vez que essa abordagem efetivamente remove as singularidades apresentadas pelas funções de Green espaciais [14]. 


\section{TEORIA}

A Figural apresenta a geometria da antena de abertura a ser analisada. A estrutura é composta por três camadas dielétricas lineares, homogèneas e anisotrópicas (anisotropias do tipo uniaxial: eixo óptico ao longo do eixo z). Dessas, duas camadas estão confinadas entre os planos condutores perfeitos $z=-d_{1}$ e $z=0$. A terceira camada está

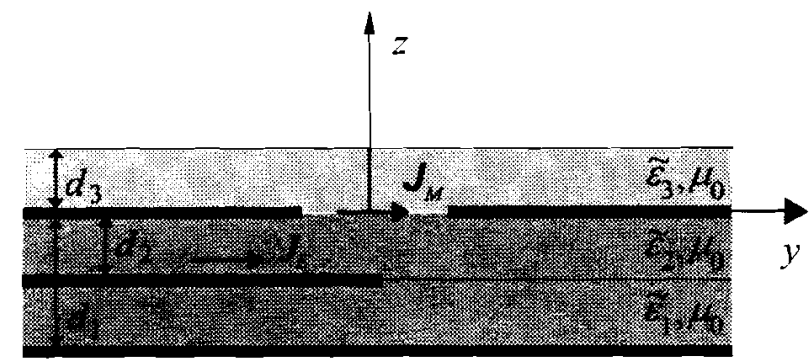

Figura 1. Antena de abertura alimentada por uma linha de fita.

localizada entre o plano $z=0$ e a interface $z=d_{3}$. Para $z>d_{3}$ tem-se o espaço livre, com permissividade elétrica $\varepsilon_{0}$ e permeabilidade magnética $\mu_{0}$. No plano de terra $(z=0)$ existe uma abertura que acopla as camadas confinadas com as camadas localizadas na região $z>0$. Cada camada uniaxial é caracterizada por uma permissividade elétrica tensorial $\widetilde{\varepsilon}_{n}$ (o indice $n=1,2$ ou 3 identifica cada uma das camadas anisotrópicas) e por uma permeabilidade magnética escalar $\mu_{0}$. Na interface $z=-d_{2}$ encontra-se impressa uma fita condutora perfeita, de espessura infinitesimal, sobre a qual é definida uma densidade de corrente elétrica superficial $\boldsymbol{J}_{E}(x, y)=J_{E x}(x, y) \boldsymbol{x}+J_{E y}(x, y) \boldsymbol{y}$ (grandezas vetoriais em negrito). Os campos eletromagnéticos nas várias camadas anisotrópicas e no espaço livre são determinados considerando-se a estrutura como um problema de contorno, onde a densidade superficial de corrente elétrica $\boldsymbol{J}_{E}(x, y)$ e a densidade superficial de corrente magnética $\boldsymbol{J}_{M}(x, y)=\boldsymbol{z} \times \boldsymbol{E}_{a}(x, y)$, onde $\boldsymbol{E}_{a}(x, y)$, é a componente tangencial do campo elétrico ao longo do plano da abertura, são as fontes virtuais desses campos $[15,16]$. Partindo das equações de Maxwell, inicialmente são determinadas as equações de onda nas camadas anisotrópicas e no espaço livre. Em seguida, tais equações são resolvidas no domínio de Fourier. Isto feito, são aplicadas as condições de contorno para os campos eletromagnéticos ao longo das interfaces $z=0, z=-d_{1}, z=-d_{2}$ e $z=d_{3}$, resultando em um sistema de quatorze equações com igual número de incógnitas. Resolvendo-se esse sistema, expressões analíticas para os campos transformados são univocamente determinadas para qualquer ponto da região $z \geq-d_{1}$. Uma vez que os cálculos são realizados no domínio de Fourier, expressões compactas, fechadas e descritas por simples funções trigonométricas são obtidas para as funções de Green espectrais. Os campos no domínio espacial são determinados através da transformada inversa de Fourier dos campos transformados. Expressões para as componentes dos campos eletromagnéticos transformados e para as funções de Green espectrais podem ser encontradas em [15], bem como os resultados mais relevantes da análise dessas funçôes.

\section{GEOMETRIA DA ANTENA DE FENDA}

A vista em planta da antena em questão é apresentada na Figura 2. A fenda, de dimensões $W_{S}$ na direção $y$ e $L_{S}$ na direção $x$, com $L_{S} \gg W_{S}$, é alimentada por uma linha de fita de dimensões $W_{L}$ na direção $x$ e $I_{P}$ na direção $y$, onde

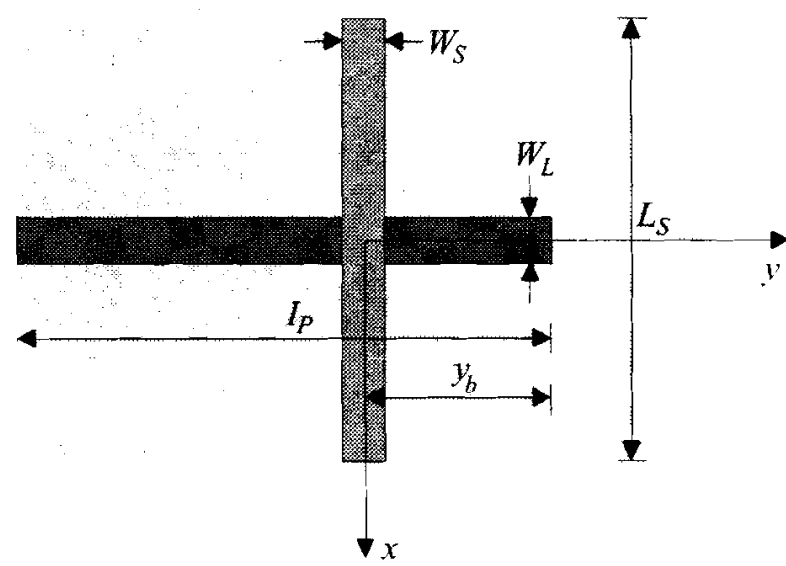

Figura 2. Vista em planta da fenda retangular alimentada por uma linha de fita.

$I_{P} \gg W_{L}$. As espessuras das camadas confinadas são $d_{2} \mathrm{e}$ $\left(d_{1}-d_{2}\right)$, enquanto que a da camada de cobertura é $d_{3}$ (Figura 1). Sendo $L_{S} \gg W_{S}$, então a ressonância da fenda é controlada pela dimensão $L_{S}$. Por outro lado, como as dimensões da linha de alimentação são tais que $I_{P} \gg W_{L}$, a propagação ocorrerá ao longo da direção $y$. Nessas condiçôes, as componentes transversais das densidades superficiais de corrente elétrica e magnética $\left(J_{E x}(x, y)\right.$ e $\left.J_{M y}(x, y)\right)$ podem ser desprezadas, de modo que $\boldsymbol{J}_{E}(x, y)=J_{E}(x, y) \boldsymbol{y}$ sobre a linha de alimentação e $\boldsymbol{J}_{M}(x, y)=J_{M}(x, y) \boldsymbol{x}$ ao longo da fenda retangular. Observe que os índices $x$ e $y$ foram eliminados das duas últimas equações com o objetivo de simplificar a nomenclatura.

\section{MÉTODO DOS MOMENTOS}

Seguindo o procedimento usual da aplicação do método dos momentos $(M o M)$ no domínio espectral $[6,10,15]$, são obtidas as seguintes equações integrais acopladas:

$$
\begin{aligned}
& \int_{-\infty}^{+\infty} \int_{y y 11} j_{E} j_{E p}^{*} d k_{x} d k_{y} \\
& +\int_{-\infty}^{+\infty} \int G_{y x m 1} j_{M} j_{E p}^{*} d k_{x} d k_{y}=0 \\
& \int_{-\infty}^{+\infty} \int_{y x m 1} j_{E} j_{M q}^{*} d k_{x} d k_{y} \\
& +\int_{-\infty}^{+\infty} \int\left(R_{x x m 2}-R_{x x m 3}\right) j_{M} j_{M q}^{*} d k_{x} d k_{y}=0
\end{aligned}
$$

em que $k_{y}$, são as denominadas variáveis espectrais, $j_{E p}^{*}$ é 
o complexo conjugado da função de teste $j_{E p}=j_{E p}\left(k_{x}, k_{1}\right)$, $j^{*}$ Mq é o complexo conjugado da função de teste $j_{N q q}=j_{N q}\left(k_{x}, k_{1}\right)$, ambas no domínio transformado, e $j_{E}=$ $j_{k}\left(k_{x}, k_{y}\right)$ e $j_{M^{\prime}}=j_{M}\left(k_{x}, k_{y}\right)$ são as transformadas de Fourier de $J_{E}(x, y)$ e $J_{M}(x, y)$, respectivamente, e:

$$
\begin{aligned}
& G_{y, 11}=\frac{-i \gamma_{21} \gamma_{22} k_{y}^{2} \operatorname{sen}\left(\gamma_{22} d_{2}\right) \operatorname{sen}\left[\gamma_{21}\left(d_{1}-d_{2}\right)\right]}{\omega u^{2} T M_{G}} \\
& -\frac{i \omega \mu_{0} k_{x}^{2} \operatorname{sen}\left(\gamma_{12} d_{2}\right) \operatorname{sen}\left[\gamma_{11}\left(d_{1}-d_{2}\right)\right]}{u^{2} T E_{G}}, \\
& G_{y x m 1}=\frac{\varepsilon_{x 2} \gamma_{21} k_{y}^{2} \operatorname{sen}\left[\gamma_{21}\left(d_{1}-d_{2}\right)\right]}{u^{2} T M_{G}} \\
& +\frac{\gamma_{12} k_{x}^{2} \operatorname{sen}\left[\gamma_{11}\left(d_{1}-d_{2}\right)\right]}{u^{2} T E_{G}}, \\
& R_{x x m 2}=\frac{i \omega \varepsilon_{x 2} k_{y}^{2}}{\gamma_{22} u^{2} T M_{G}}\left\{\varepsilon_{x 2} \gamma_{21} \operatorname{sen}\left[\gamma_{21}\left(d_{1}-d_{2}\right)\right]\right. \\
& \left.\cdot \operatorname{sen}\left(\gamma_{22} d_{2}\right)-\varepsilon_{x 1} \gamma_{22} \cos \left[\gamma_{21}\left(d_{1}-d_{2}\right)\right] \cos \left(\gamma_{22} d_{2}\right)\right\} \\
& +\frac{i \gamma_{12} k_{x}^{2}}{\omega \mu_{0} u^{2} T E_{G}}\left\{\gamma_{12} \operatorname{sen}\left[\gamma_{11}\left(d_{1}-d_{2}\right)\right] \operatorname{sen}\left(\gamma_{12} d_{2}\right)\right. \\
& \left.-\gamma_{11} \cos \left[\gamma_{11}\left(d_{1}-d_{2}\right)\right] \cos \left(\gamma_{12} d_{2}\right)\right\} \text {, } \\
& R_{x x m 3}=\frac{\omega \varepsilon_{x 3} k_{y}^{2}}{\gamma_{23} u^{2} T M_{S}}\left\{-\varepsilon_{x 3} \gamma_{0} \operatorname{sen}\left(\gamma_{23} d_{3}\right)\right. \\
& \left.+i \varepsilon_{0} \gamma_{23} \cos \left(\gamma_{23} d_{3}\right)\right\}+\frac{\gamma_{13} k_{x}^{2}}{\omega \mu_{0} u^{2} T E_{S}} \\
& \cdot\left\{-\gamma_{13} \operatorname{sen}\left(\gamma_{13} d_{3}\right)+i \gamma_{0} \cos \left(\gamma_{13} d_{3}\right)\right\}, \\
& T M_{G}=\varepsilon_{x 1} \gamma_{22} \operatorname{sen}\left(\gamma_{22} d_{2}\right) \cos \left[\gamma_{21}\left(d_{1}-d_{2}\right)\right] \\
& +\varepsilon_{x 2} \gamma_{21} \cos \left(\gamma_{22} d_{2}\right) \operatorname{sen}\left[\gamma_{21}\left(d_{1}-d_{2}\right)\right] \\
& T E_{G}=\gamma_{11} \operatorname{sen}\left(\gamma_{12} d_{2}\right) \cos \left[\gamma_{11}\left(d_{1}-d_{2}\right)\right] \\
& +\gamma_{12} \cos \left(\gamma_{12} d_{2}\right) \operatorname{sen}\left[\gamma_{11}\left(d_{1}-d_{2}\right)\right] \text {, } \\
& T M_{S}=\varepsilon_{0} \gamma_{23} \operatorname{sen}\left(\gamma_{23} d_{3}\right)-i \varepsilon_{x 3} \gamma_{0} \cos \left(\gamma_{23} d_{3}\right), \\
& T E_{S}=\gamma_{0} \operatorname{sen}\left(\gamma_{13} d_{3}\right)-i \gamma_{13} \cos \left(\gamma_{13} d_{3}\right), \\
& \gamma_{1 n}=\sqrt{\omega^{2} \mu_{0} \varepsilon_{x n}-u^{2}}, \quad ; \operatorname{Im}\left[\gamma_{1 n}\right] \leq 0, \\
& \gamma_{2 n}=\sqrt{\frac{\varepsilon_{x n}}{\varepsilon_{z n}}\left(\omega^{2} \mu_{0} \varepsilon_{z n}-u^{2}\right)} \quad ; \operatorname{Im}\left[\gamma_{2 n}\right] \leq 0, \\
& \gamma_{0}=\sqrt{k_{0}^{2}+u^{2}}, \quad ; \operatorname{Im}\left[\gamma_{0}\right] \leq 0, \\
& k_{0}^{2}=\omega^{2} \mu_{0} \varepsilon_{0}, \\
& u^{2}=k_{x}^{2}+k_{v}^{2} .
\end{aligned}
$$

Nessa formulação, a estrutura é excitada pela densidade de corrente elétrica incidente que se encontra incorporada a $j_{E}$.

\subsection{EXPANSÃO DAS DENSIDADES DE COR- RENTE ELÉTRICA E MAGNÉTICA}

Considera-se, neste trabalho, que a linha de alimentação suporta o modo fundamental de propagação que é o caso usual de interesse prático. Dessa forma, os efeitos produzidos pela fenda e pela terminação abrupta da linha, sobre a densidade superficial de corrente elétrica, são computados no coeficiente de reflexão da corrente. Também é conveniente considerar essa densidade de corrente como o produto de duas funções independentes de $x$ e $y$, de modo que $J_{E}(x, y)=J_{E x}(x) J_{E y}(y)[15,17]$. Além disso, a expansão da função densidade superficial de corrente elétrica sobre a linha de alimentação, em termos de funções de base conhecidas, deve levar em conta o fato dessa densidade de corrente tender a infinito nas bordas localizadas em $x=-W_{L} / 2$ e em $x=+W_{L} / 2$, denominado condiçâo de borda. Ao longo da direção $y$, a função de base será descrita pela superposição de funções de onda incidente e refletida, no modo fundamental de propagação, além de um número $M_{y}$ de funções triangulares definidas a partir do extremo da linha de forma a representar a corrente que, nesta região, tem um comportamento que se afasta do modo fundamental [17]. Assim, ao longo da direção $x$, a função densidade superficial de corrente será expressa por:

$$
J_{E x}(x)=\left\{\begin{array}{cl}
\frac{1}{\sqrt{1-\left(2 x / W_{L}\right)^{2}}} ; & -W_{L} / 2<x<+W_{L} / 2, \\
0 & ; \text { caso contrário },
\end{array}\right.
$$

cuja transformada de Fourier é dada por:

$$
j_{E x}\left(k_{x}\right)=\pi \frac{W_{L}}{2} J_{0}\left(k_{x} W_{L} / 2\right),
$$

onde $J_{0}(z)$ é a função de Bessel de primeira espécie e ordem zero.

Ao longo da direção $y$, a função densidade superficial de corrente será descrita por:

$$
J_{E y}(y)=J_{E y}^{\prime}(y)+J_{E y}{ }^{R}(y)+\sum_{m=1}^{M_{y}} I_{E m} J_{E m y}(y),
$$

onde

$$
J_{E y}^{l}(y)=e^{-i k_{e}\left(y-y_{b}\right)},
$$

representa a densidade superficial de corrente associada à onda incidente $\mathrm{e}$,

$$
J_{E y}^{R}(y)=-\Gamma e^{i k_{e}\left(y-y_{b}\right)},
$$

representa a densidade superficial de corrente associada à onda refletida. A constante de propagação do modo funda- 
mental da linha de transmissão é $k_{e}$, sendo $I_{E m}$ coeficientes numéricos a serem determinados. Note que o coeficiente de reflexão $\Gamma$ foi definido no extremo da linha, isto é, em $y=y_{b}$.

Para o conjunto de funções triangulares optou-se pela seguinte expressão:

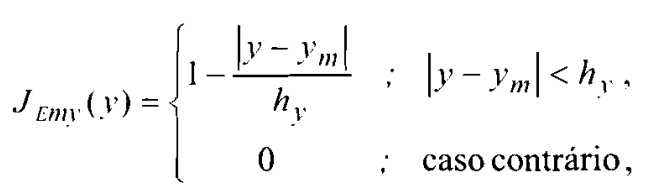

onde $y_{m}=y_{b}-m h_{y}$ e $h_{y}=L_{L} /\left(M_{y}+1\right)$.

Na Figura 3 são apresentados os gráficos dos modos de expansão da densidade superficial de corrente elétrica $J_{E v} v(y)$, onde se observa que o domínio da função triangular é $L_{L}$. Uma vez que tanto a função seno como a triangular se anulam em $y=y_{b}$, para manter a condição de corrente elétrica nula na extremidade da linha de alimentação, a função co-seno foi truncada em $v_{b}-\pi / 2 k_{e}$, de acordo com os critérios apresentados em [17].

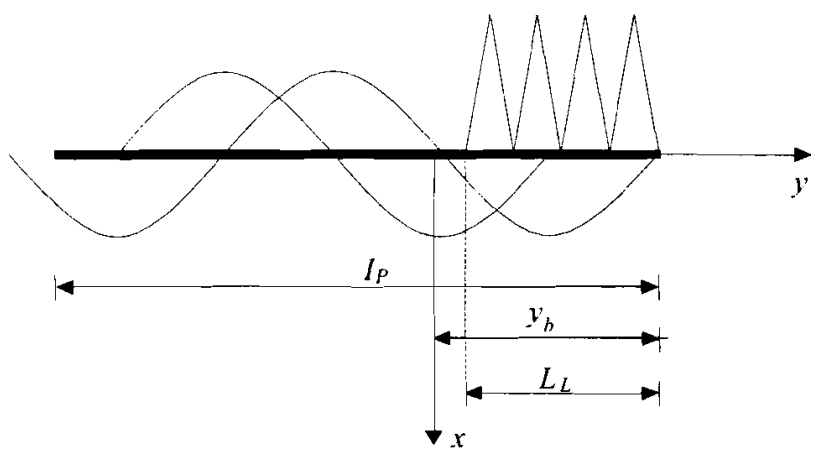

Figura 3. Modos de expansão da densidade de corrente $J_{E y}(y)$, sobre a linha de alimentação.

Considerando um número inteiro e par de ciclos (v) para as funções seno e co-seno, suas transformadas de Fourier são determinadas por:

$$
f_{c}\left(k_{y}\right)=\int_{\left(y_{b}-\pi(2 v+1) / 2 k_{e}\right)}^{\left(y_{b}-\pi / 2 k_{\xi^{\prime}}\right)} \cos \left[k_{e}\left(y-y_{b}\right)\right] e^{i k_{y} \cdot y} d y,
$$

e

$$
f_{s}\left(k_{y}\right)=\int_{\left(y_{b}-v \pi / k_{k}\right)}^{v_{b}} \operatorname{sen}\left[k_{e}\left(y-y_{b}\right)\right] e^{i k_{y} y} d y .
$$

Realizadas as integrações, os resultados são agrupados convenientemente nas formas seguintes:

$$
\begin{aligned}
& f_{a}\left(k_{y}\right)=f_{c}\left(k_{y}\right)+i f_{s}\left(k_{y}\right) \\
& =\frac{k_{e} e^{i k_{y} v_{b}}}{k_{e}^{2}-k_{y}^{2}}\left(e^{-i v \pi k_{y} / k_{e}}-1\right)\left(e^{-i \pi k_{y} / 2 k_{e}}+i\right) .
\end{aligned}
$$

e

$$
f_{d}\left(k_{y}\right)=f_{c}\left(k_{y}\right)-i f_{s}\left(k_{y}\right)
$$

$$
=\frac{k_{e} e^{i k_{y} y_{h}}}{k_{e}^{2}-k_{y}^{2}}\left(e^{-i v \pi k_{y} / k_{e}}-1\right)\left(e^{-i \pi k_{y} / 2 k_{e}}-i\right) .
$$

Calculando-se a transformada de Fourier da função de base triangular, tem-se:

$$
j_{E m y}\left(k_{y}\right)=h_{y} \frac{\operatorname{sen}^{2}\left(k_{y}, h_{y} / 2\right)}{\left(k_{y}, h_{y} / 2\right)^{2}} e^{i k_{y} y_{m}} .
$$

No caso da densidade superficial de corrente magnética, a expansão é realizada em termos de $N_{x}$ funçôes triangulares com condiçôes de borda, similares às descritas anteriormente pelas equações (16) e (21).

Como funções de teste, foram utilizadas funções triangulares ao longo da direção $y$, no caso da linha, e ao longo da direção $x$, no caso da fenda. Ao longo da direção $x$, no caso da linha, e ao longo da direção $y$, no caso da fenda, foram utilizadas funções que levam em conta a condição de borda. Assim, no domínio transformado, as funções de teste são escritas como:

$$
\begin{aligned}
& j_{E p x}\left(k_{x}\right)=\pi \frac{W_{L}}{2} J_{\mathrm{o}}\left(k_{x} W_{L} / 2\right), \\
& j_{E p y^{\prime}}\left(k_{y}\right)=h_{y} \frac{\operatorname{sen}^{2}\left(k_{y} h_{y} / 2\right)}{\left(k_{y} h_{y} / 2\right)^{2}} e^{i k_{y} y_{p}}, \\
& j_{M q x}\left(k_{x}\right)=h_{x} \frac{\operatorname{sen}^{2}\left(k_{x} h_{x} / 2\right)}{\left(k_{x} h_{x} / 2\right)^{2}} e^{i k_{x} x_{q}}, \\
& j_{M q y}\left(k_{y}\right)=\pi \frac{W_{S}}{2} J_{\mathrm{o}}\left(k_{y} W_{S} / 2\right) .
\end{aligned}
$$

\subsection{SISTEMA DE EQUAÇÕES}

Com as funções de base e de teste definidas é possivel montar o seguinte sistema de $\left(M_{y}+N_{x}+1\right)$ equações na forma matricial [15]:

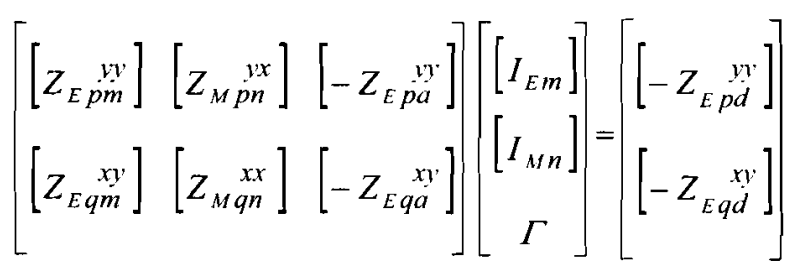

com $p=1, \ldots,\left(M_{y}+1\right)$ e $q=1, \ldots, N_{x}, I_{M n}$ são coeficientes numéricos relacionados com a expansão da densidade superficial de corrente magnética (a serem determinados). $\mathrm{Na}$ solução deste sistema, depara-se com o cálculo de integrais que, em geral, são numericamente ineficientes. Para aumentar a eficiência desses cálculos são empregadas técnicas de redução ao primeiro quadrante, através do estudo da paridade de seus integrandos, de mudança de variável, com o objetivo de reduzir uma das integrais a limites finitos $\left(k_{x}=\beta \cos \alpha\right.$ e $k_{y}=\beta$ sen $\left.\alpha\right)$ e de subtração do termo dominante, para acelerar a convergência das 
integrais. Como um exemplo, a expressão para o cálculo de $Z_{\text {Eqm }}^{x y}$ é apresentada abaixo:

$$
\begin{aligned}
Z_{E: q m}^{x y}= & \frac{16}{h_{x} h_{y}} \int_{0}^{+\infty} \int_{0}^{\pi / 2}\left[\frac{\varepsilon_{x 2} \gamma_{21}}{\operatorname{sen}\left(\gamma_{22} d_{2}\right) T_{1}} \operatorname{sen}^{2} \alpha\right. \\
& \left.+\frac{\gamma_{12}}{\operatorname{sen}\left(\gamma_{12} d_{2}\right) T_{2}} \cos ^{2} \alpha\right] B_{E q m}^{x y} d \alpha d \beta
\end{aligned}
$$

onde

$$
\begin{aligned}
B_{E q m} \underset{m}{x}= & \frac{j_{E x} j_{M y} \operatorname{sen}^{2}\left(h_{x} \beta \operatorname{sen} \alpha / 2\right) \operatorname{sen}^{2}\left(h_{y} \beta \operatorname{sen} \alpha / 2\right)}{\beta^{3} \cos ^{2} \alpha \operatorname{sen}^{2} \alpha} \\
& \quad \cdot \cos \left(x_{q} \beta \cos \alpha\right) \cos \left(y_{m} \beta \operatorname{sen} \alpha\right) \\
T_{1}= & \varepsilon_{x 2} \gamma_{21} \operatorname{cotg}\left(\gamma_{22} d_{2}\right) \\
& +\varepsilon_{x \mid} \gamma_{22} \operatorname{cotg}\left[\gamma_{21}\left(d_{1}-d_{2}\right)\right] \\
T_{2}= & \gamma_{12} \operatorname{cotg}\left(\gamma_{12} d_{2}\right)+\gamma_{11} \operatorname{cotg}\left[\gamma_{11}\left(d_{1}-d_{2}\right)\right]
\end{aligned}
$$

Conhecidos os parâmetros $k_{e}, Z_{\mathrm{o}}$ e $\Gamma$, a impedância de entrada da antena de fenda pode ser obtida da seguinte expressão:

$$
Z_{i n}(y)=Z_{\mathrm{o}} \frac{1+\Gamma e^{2 i k_{c} \cdot !}}{1-\Gamma e^{2 i k_{c^{\prime} \cdot}}}
$$

$\mathrm{O}$ procedimento utilizado no cálculo da constante de propagação $k_{e}$ já foi discutido em [16]. O da impedância característica $Z_{0}$, considera a linha infinita e operando no modo fundamental quase-TEM, de modo que,

$$
Z_{\mathrm{o}}=\frac{V}{I}
$$

onde a tensão em um determinado plano normal à direção de propagação, entre a fita condutora e um dos planos de terra, pode ser calculada pela integral do campo elétrico ao longo do eixo $z$, tanto no meio 1 como no meio 2 , e a corrente, neste mesmo plano, pela integral da densidade de corrente elétrica sobre a fita condutora. Por exemplo, se o cálculo for realizado entre os pontos $\left(x_{0}, y_{0},-d_{2}\right) \mathrm{e}$ $\left(x_{0}, y_{0},-d_{1}\right)$, a equação (37) é reescrita da seguinte forma:

$$
Z_{0}=\frac{1}{\pi} \int_{-d_{2}}^{-d_{1}} \int_{0}^{+\infty} G_{z \cdot 11}\left(k_{x}, k_{e}, z\right) J_{0}\left(k_{x} W_{L} / 2\right) e^{-i k_{x} x_{0}} d k_{x} d z
$$

e

$G_{z y 111}\left(k_{x}, k_{y}, z\right)=\frac{-\varepsilon_{x 1} \gamma_{22} \operatorname{sen}\left(\gamma_{22} d_{2}\right) \cos \left[\gamma_{21}\left(d_{1}+z\right)\right] k_{y}}{\omega \varepsilon_{z 1} T M_{G}}$

\section{RESULTADOS NUMÉRICOS}

Considerando a teoria acima exposta, efeitos da anisotropia uniaxial sobre a constante de propagação $k_{e}$, a impedân- cia característica $Z_{\mathrm{o}}$ e a impedância de entrada da antena de fenda, foram analisados. Inicialmente, nas Figuras 5 a 8 são apresentadas curvas de dispersão para a constante de propagação e para a impedância característica da linha de fita descrita na Figura 4, calculadas com $d_{1}=3,524 \mathrm{~mm}$, $d_{2}=1,524 \mathrm{~mm}$ e $W_{L}=5,6 \mathrm{~mm}$, tendo o vácuo como o dielé-

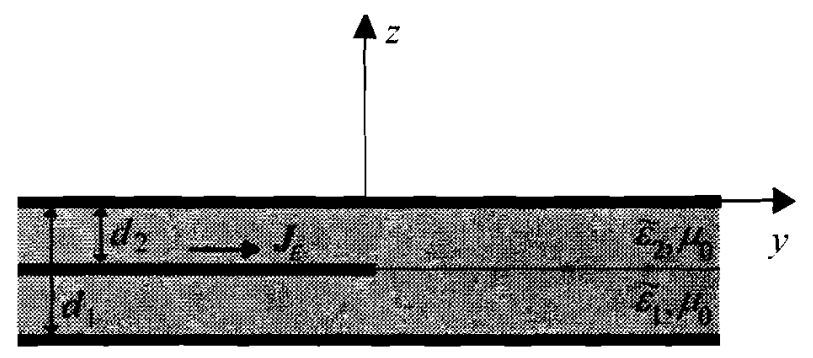

Figura 4. Linha de fita com substratos anisotrópicos.

trico da camada 1. Razões entre permissividades na direção $z$ e permissividades na direção $x, \varepsilon_{z} / \varepsilon_{x}$, iguais a 1,$0 ; 1,1 ; 1,2$ e 1,3, foram consideradas para o dielétrico da camada 2 . Em todos esses casos utilizou-se a permissividade relativa na direção $x$, da camada 2, como sendo 2,55. Observa-se das curvas da Figura 5 que, para uma dada freqüencia, $k_{\mathfrak{e}}$ aumenta com o aumento da razão de anisotropia $\varepsilon_{z} / \varepsilon_{x}$. Por exemplo, para a freqüência de $2 \mathrm{GHz}, k_{\mathfrak{c}^{\prime}}$ aumenta de aproximadamente $2,1 \mathrm{rad} / \mathrm{m}$, para cada incremento de 0,1 na razào de anisotropia. Para melhor visualizar esta dependência da constante de propagação com a razão de anisotropia, gráficos calculados na freqüência de $2 \mathrm{GHz}$ são apresentados na Figura 6.

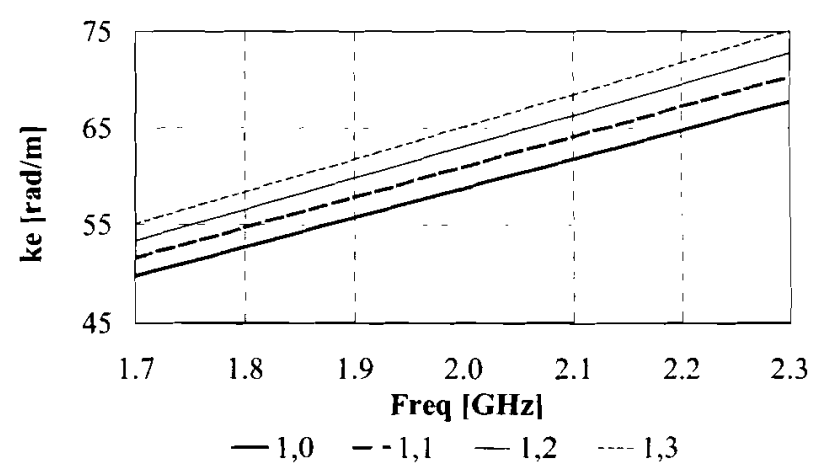

Figura 5. Curvas de dispersão da constante de propagação para diferentes razões de anisotropia.

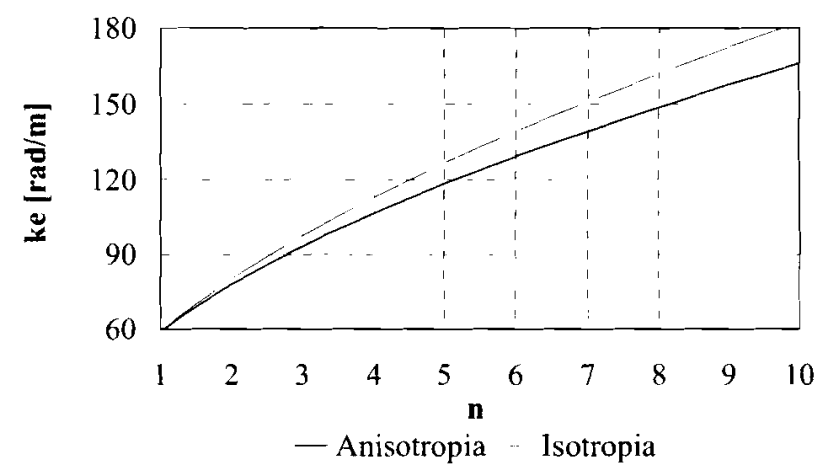

Figura 6. Constante de propagação em função da razão de anisotropia na frequiência de $2 \mathrm{GHz}$. 
Quando o substrato da camada 2 é anisotrópico, o indice n representa a razão $\varepsilon_{=} / \varepsilon_{x}$, com a permissividade relativa $\varepsilon_{r x}$ constante e igual a 2,55. Para o caso em que o substrato da camada 2 é isotrópico, o índice $\mathbf{n}$ indica um fator que multiplica a constante 2,55 . O resultado desse produto será a permissividade relativa da camada 2 . Por exemplo, para $\mathbf{n}$ igual a 3 , a constante de propagação para a condição anisotrópica foi calculada com $\varepsilon_{r: x}$ igual a 2,55 e $\varepsilon_{r=}$ igual a 7,65 , enquanto que a do meio isotrópico $\operatorname{com} \varepsilon_{r: x}=\varepsilon_{r=}=$ 7,65. Portanto, o fato da constante relativa $\varepsilon_{r}$. ser menor que a da direção $z$, provocou um decréscimo no valor de $k_{e}$. Quanto menor for $\varepsilon_{r x}$, com relação a um determinado valor de $\varepsilon_{r z}$, mais a curva anisotrópica se afasta da isotrópica, calculada para $\varepsilon_{r}=\varepsilon_{r z}$

$\mathrm{Na}$ Figura 7 são apresentados os resultados para a impedância característica da referida linha. Comportamento semelhante ao observado nas curvas da constante de propagação $k_{e}$ se repete neste caso. Por exemplo, para a freqüência de $2 \mathrm{GHz}$, o valor de $Z_{0}$ varia aproximadamente

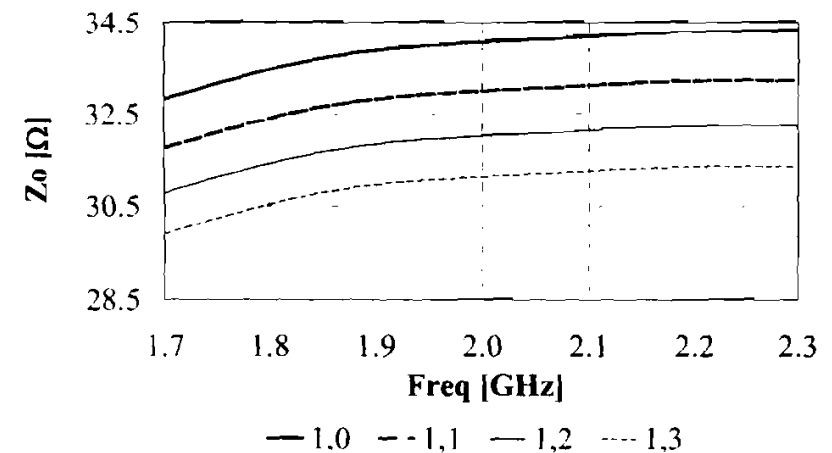

Figura 7. Curvas da impedância característica para diferentes razões de anisotropia.

de $1 \Omega$, na medida em que a razão de anisotropia aumenta de 0,1 . Para melhor visualizar a dependencia da impedância característica com a razão de anisotropia, gráficos calculados na frequêencia de $2 \mathrm{GHz}$ são apresentados na Figura 8.

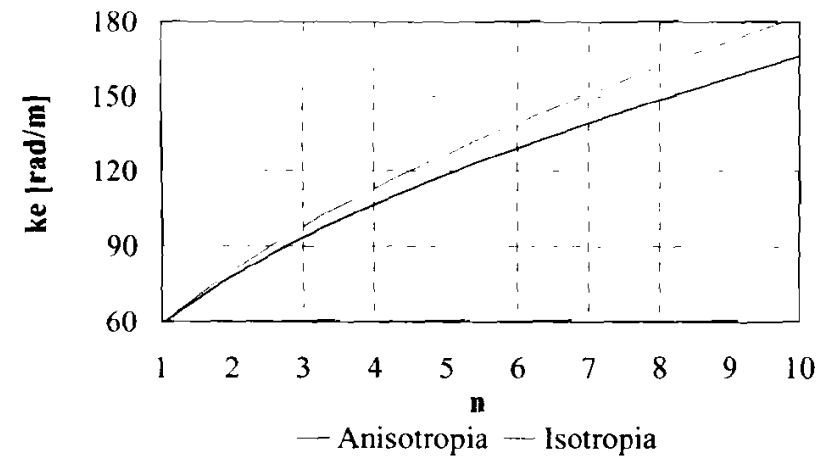

Figura 8. Impedância característica em função da razão de anisotropia na freqüência de $2 \mathrm{GHz}$.

Na Tabela 1 são apresentados resultados para a impedância característica de linhas de fita uniformes $\left(d_{1}=2 d_{2}=\right.$ $4 \mathrm{~mm}$ ) com substratos isotrópicos idênticos, isto é, quando se utiliza a teoria uniaxial na condição particular (tucp) definida por $\varepsilon_{r \times 1}=\varepsilon_{r z 1}=\varepsilon_{r \times 2}=\varepsilon_{r z 2}=\varepsilon_{r}$, em comparação com os calculados pelos programas TLINE [18] e ENSEMBLE
[19]. Nota-se uma boa concordància entre esses resultados.

\begin{tabular}{ccccc}
\hline$\varepsilon_{r}$ & $\begin{array}{c}W_{L} \\
(\mathrm{~mm})\end{array}$ & $\begin{array}{c}\text { tucp } \\
2 \mathrm{GHz}\end{array}$ & TLINE & $\begin{array}{c}\text { ENSEMBLE } \\
2 \mathrm{GHz}\end{array}$ \\
\hline 1,0 & 2,0 & $97,7 \Omega$ & $100,1 \Omega$ & $101,7 \Omega$ \\
1,0 & 3,0 & $77,0 \Omega$ & $79,1 \Omega$ & $80,2 \Omega$ \\
1,0 & 4,0 & $63,1 \Omega$ & $65,4 \Omega$ & $66,3 \Omega$ \\
2,55 & 2,0 & $61,1 \Omega$ & $62,7 \Omega$ & $63,6 \Omega$ \\
2.55 & 3,0 & $48,9 \Omega$ & $49,5 \Omega$ & $50,2 \Omega$ \\
2,55 & 4,0 & $40,1 \Omega$ & $41,0 \Omega$ & $41,5 \Omega$ \\
\hline
\end{tabular}

Tabela 1. Impedância característica de linhas de fita uniformes com substrato dielétricos iguais.

Efeitos da razão de anisotropia $\varepsilon_{\varepsilon} / \varepsilon_{X}$ na impedancia de entrada da antena de fenda serão discutidos a seguir. Com essa finalidade, define-se uma antena Teste com características elétricas e dimensões geométricas descritas na Tabela 2. Na Figura 9 são apresentadas curvas para a impedância de entrada normalizada $\left(z_{\text {in }}=Z_{\text {in }} / Z_{\mathrm{o}}\right)$ da antena de fenda, calculadas com as dimensões geométricas da antena Teste. tendo o vácuo como o dielétrico da camada 1 . Razões de anisotropia $\varepsilon_{z} / \varepsilon_{\mathrm{x}}$ iguais a 1,$0 ; 1,1 ; 1,2$ e 1,3 foram consideradas para os dielétricos das camadas 2 e 3 . Em todos esses casos utilizou-se a permissividade relativa na direção $x$, das camadas 2 e 3, como sendo 2,55 e uma tangente de perdas, para ambos os meios, igual a 0,0018 . Nota-se destas curvas que o aumento da razão de anisotropia $\varepsilon_{z} / \varepsilon_{x}$ provocou o deslocamento da ressonância da antena para valores mais baixos que o obtido para a condição isotrópica. Além disso, a forma da impedância de entrada é modificada na medida em que a razão de anisotropia aumenta.

\begin{tabular}{|c|c|c|c|}
\hline CAMADA & $\operatorname{Re}\left[\varepsilon_{r}\right]$ & $\begin{array}{l}\text { TANGENTE } \\
\text { DE PERDAS }\end{array}$ & $\begin{array}{l}\text { ESPESSURA } \\
(\mathrm{mm})\end{array}$ \\
\hline 1 & 1,0 & 0,0 & 2,0 \\
\hline 2 & 2,55 & 0,0018 & 1,524 \\
\hline 3 & 2,55 & 0,0018 & 1,524 \\
\hline \multicolumn{4}{|c|}{ DiMENSŌES (mm) } \\
\hline \multicolumn{2}{|c|}{$L_{S}$} & \multicolumn{2}{|c|}{41,0} \\
\hline \multicolumn{2}{|c|}{$W_{S}$} & \multicolumn{2}{|c|}{4,0} \\
\hline \multicolumn{2}{|c|}{$y_{b}$} & \multicolumn{2}{|c|}{8,8} \\
\hline \multicolumn{2}{|c|}{$I_{P}$} & \multicolumn{2}{|c|}{110,55} \\
\hline \multicolumn{2}{|c|}{$W_{L}$} & \multicolumn{2}{|c|}{5,6} \\
\hline
\end{tabular}

Tabela 2. Características elétricas e dimensões geométricas da antena Teste.

Na Figura 10 são apresentados resultados da impedância de entrada normalizada para a antena Teste, calculados com a teoria uniaxial na condição isotrópica (tuci) com 40 funçōes triangulares sobre a linha de alimentação, 10 funções do mesmo tipo sobre a fenda e $L_{L}=164 \mathrm{~mm}$, cujos parâmetros estão definida na Tabela 2, em comparação com os obtidos pelo programa ENSEMBLE. Observa-se uma boa concordância entre esses resultados. 


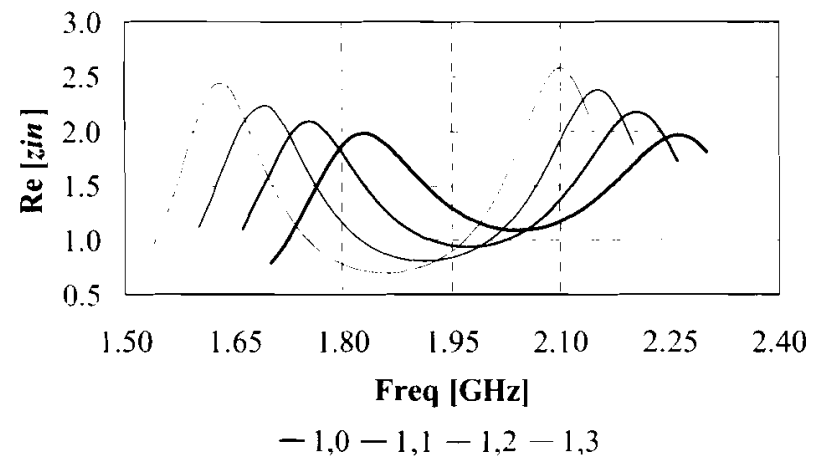

(a)

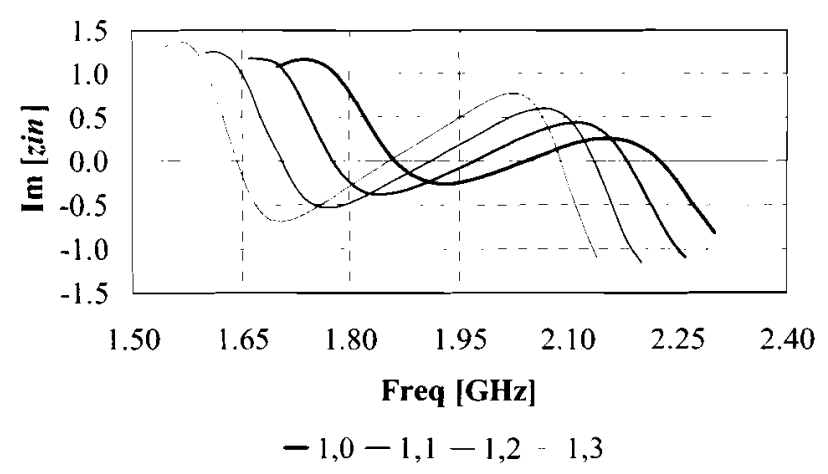

(b)

Figura 9. Efeitos da razão axial sobre a impedância de entrada normalizada da antena de fenda

(a) $\operatorname{Re}\left[z_{i n}\right]$ (b) $\operatorname{Im}\left[z_{i n}\right]$

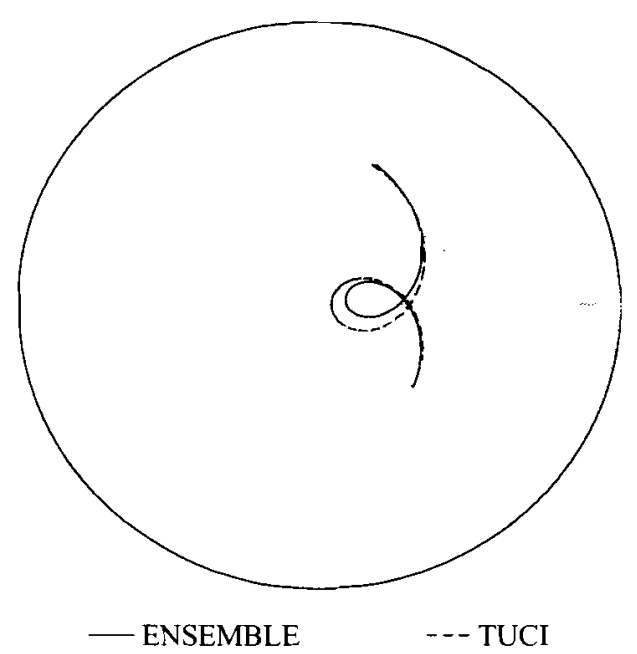

Figura 10. Impedância de entrada normalizada da antena Teste sobre a carta de Smith.

Uma vez determinados os coeficientes da expansão das funções de base para as densidades superficiais de corrente elétrica e magnética, é possível o cálculo essas densidades de corrente. Nas Figuras 11 e 12 são apresentados, em gráficos tridimensionais, os resultados obtidos para as densidades superficiais de corrente elétrica, sobre a linha de alimentação,. e magnética, sobre a fenda, calculadas na freqüência de $2 \mathrm{GHz}$ para a antena Teste na condição isotrópica. Para melhor observar o comportamento da densidade de corrente sobre a linha de alimentação, na Figura 13 é apresentado um gráfico bidimensional calculado no plano $x=0$. Observa-se desse gráfico que a onda

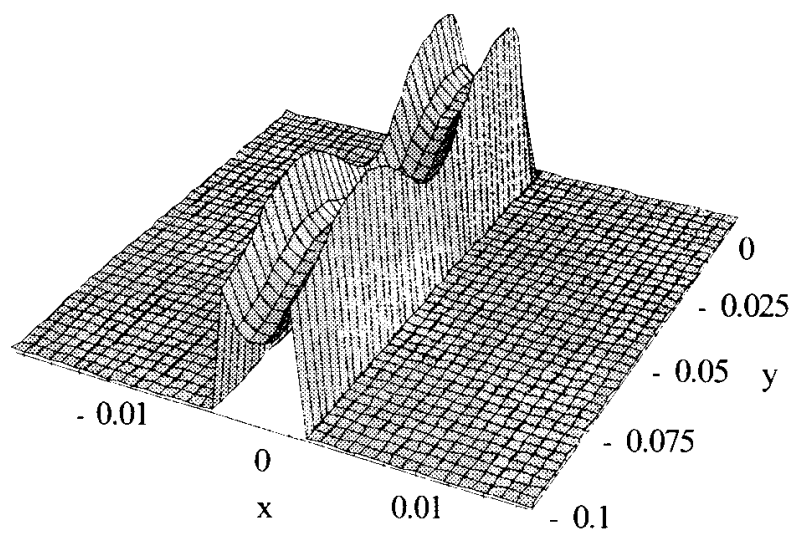

Figura 11. Gráfico 3D da densidade superficial de corrente elétrica sobre a linha de alimentação.

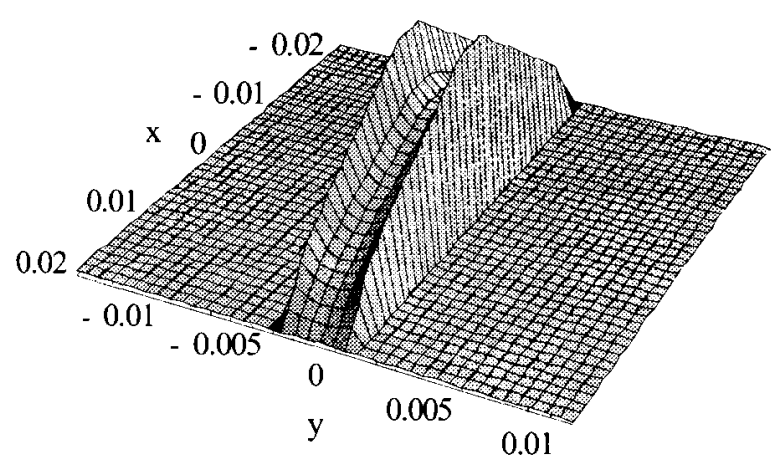

Figura 12. Gráfico 3D da densidade superficial de corrente magnética sobre a fenda.

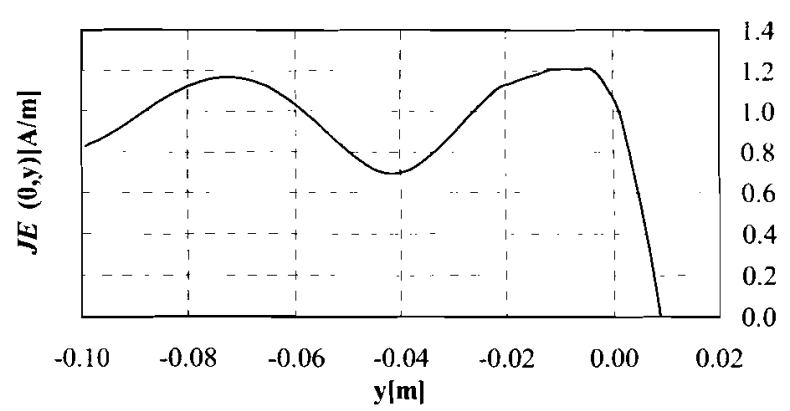

Figura 13. Gráfico 2D da densidade superficial de corrente elétrica sobre a linha de alimentação.

estacionária, na freqüência de $2 \mathrm{GHz}$, apresenta um baixo coeficiente de reflexão. Resolvendo-se o sistema (31) para a antena em questão, encontra-se que o coeficiente de reflexão, neste caso, vale: $\Gamma=0,0801-i 0,0253$ (resultando em um coeficiente de onda estacionária da ordem de 1,18 ), confirmando que a antena está adequadamente casada nesta freqüência.

\section{CONCLUSÕES}

No presente trabalho é apresentado um modelo dinâmico para análise de antenas de abertura alimentadas por linhas de fita com substratos anisotrópicos uniaxiais. Neste modelo, os campos eletromagnéticos transformados, nas diversas camadas da antena, são determinados conside- 
rando-se a estrutura como um problema de contorno onde as densidades superficiais de corrente elétrica e magnética são as fontes virtuais desses campos. Esse procedimento permite estabelecer as equações integrais que possibilitam o cálculo, via método dos momentos, das distribuições de corrente acima citadas, da impedância característica, da constante de propagação e do coeficiente de reflexão da linha de fita. Efeitos da anisotropia uniaxial sobre esses parâmetros foram discutidos. São particularmente interessantes os resultados obtidos para a impedância de entrada da antena de fenda retangular. Verificou-se que o aumento da razão de anisotropia $\left(\varepsilon_{z} / \varepsilon_{x}\right)$, além do esperado efeito sobre a freqüência de ressonância da antena, provocou uma modificação substancial na curva de impedância da referida fenda. Geometrias com substratos isotrópicos também foram analisadas. Para esse caso isotrópico, os resultados obtidos estão em boa concordância com os calculados por programas disponiveis comercialmente (esses programas não têm a capacidade de analisar estruturas com dielétricos uniaxiais).

A estrutura considerada nesse trabalho, linha de fita excitando uma fenda, pode ser utilizada no projeto de antenas ativas com feixes múltiplos, tendo como principais vantagens a inexistência de irradiação traseira e a reduzida iteração do sistema ativo de alimentação com o elemento irradiador propriamente dito.

\section{AGRADECIMENTOS}

Este trabalho foi realizado com apoio parcial da CAPES e da OACI-BRA/95/802, Objetivo 6.

\section{REFERÊNCIAS}

[1] J.C.S. Lacava, C.R. Pérez Rodrigues e C.C.M. Junqueira: "Uma solução para as redes de antenas conformáveis dos foguetes SONDA IV e VLS operarem com dois canais de telemetria em banda S," $7^{2}$ Simpósio Brasileiro de Telecomunicações, Florianópolis, Anais: 332-335, set. 1989.

[2] J.M. Bezerra e J.C.S. Lacava, "Aplicação de antenas de microlinha em telemetria de ensaios em vôo," $1 I^{\underline{o}}$ Seminário ADUNESP. Guaratinguetá, Anais, V-II: 590-594, nov. 1990.

[3] L. Cividanes, V.P.D. da Costa Jr. e J.C.S. Lacava, "Antena de microfita para microssatélite científico," $13^{\text {o }}$ Simpósio Brasileiro de Telecomunicações, Águas de Lindóia, Anais, VII: $541-545$, set. 1995.

[4] L. Cividanes, R.A.S. Araujo, J.C.S. Lacava e P.J.C. Rodrigues, "Antena de microfita ativa para aplicação em GPS," 150 Simpósio Brasileiro de Telecomunicações, Recife, Anais: 519522 , set. 1997.

[5] L. Cividanes, C.A.B. Gonçalves e J.C.S. Lacava, "Sistema de antenas de microfita com polarização circular para satélite científico: concepção e qualificação para vôo," $8^{\underline{Q}}$ Simpósio Brasileiro de Microondas e Optoeletrônica, Joinville, Anais: 224-228, jul. 1998.

[6] D.M. Pozar and D.H. Schaubert (Eds.): Microstrip antennas Piscataway: IEEE Press, 1995.

[7] N.G. Alexópoulos: "Integrated-circuit structures on anisotropic substrates," IEEE Trans. Microwave Theory Tech., vol. 33, pp. 847-881, Oct. 1985.

[8] D.M. Pozar: "Radiation and scattering from a microstrip patch on a uniaxial substrate," IEEE Trans. Antennas Propagat., vol. 35, pp. 613-621, June 1987.

[9] R.M. Nelson, D.A. Rogers and A.G. D'assunção: "Resonant frequency of a rectangular microstrip patch on several uniaxial substrates," IEEE Trans. Antennas Propagat., vol. 38. pp. $973-$ 981, July 1990.

[10] L.B.T. Cividanes, "Análise de antenas de microlinha em estruturas multicamadas com dielétricos uniaxiais," Tese de Doutorado. ITA, São José dos Campos, 1992.

[11] F. Bouttout, F. Benabdelaziz, A. Benghalia, D. Khedrouche and T. Fortaki, "Uniaxially anisotropic substrate effects on resonance of rectangular microstrip patch antenna," Electron. Lett., 1999, 35(4), pp. 255-256.

[12] M. Kahrizi, T.K. Sarkar and Z.A. Maricevic: "Analysis of a wide radiating slot in the ground plane of a microstrip line," IEEE Trans. Microwave Theory Tech., vol. 41, pp. 29-37, Jan. 1993.

[13] F. Croq: "Antennes microrubans multicouches alimentees par ouverture. Applications aux antennes a large bande et haute purete de polarisation pour radar et telecommunication spatiale. Ph.D dissertation. Universite de Nice-Sophia, Antipolis, France, Oct. 1990.

[14] C. Scott: The spectral domain method in electromagnetics. Norwood: Artech House, 1989.

[15] A.V. Proaño De la Torre: "Análise de estruturas multicamadas uniaxiais," Tese de Mestrado. ITA, São José dos Campos, 1999

[16] L. Cividanes, A.V. Proaño De la Torre e J.C.S. Lacava: "Análise de estruturas multicamadas uniaxiais," Revista da Sociedade Brasileira de Telecomunicações, vol. 15, pp. 5058 , jun. 2000.

[17] R.W. Jackson and D.M. Pozar: "Full-wave analysis of microstrip open-end and gap discontinuities," IEEE Trans. Microwave Theory Tech., vol. 33, pp. 1036-1042, Oct. 1985.

[18] D.M. Pozar - Program TLINE, in: Antenna design using personal computers. Dedham: Artech House, 1985.

[19] ENSEMBLE ${ }^{\mathrm{TM}}$ - Version 4.02, Design, review, \& ID array synthesis. Boulder Microwave Technologies, Inc., 1996.

Ana Vanessa Proaño De la Torre nasceu em Quito, Equador. em 1971. Graduou-se em Engenharia Eletrônica pela Escuela Politécnica del Ejército, Equador, em 1996. Obteve o título de Mestre em Ciências pelo Instituto Tecnológico de Aeronáutica, São José dos Campos, em 1999. Desde 1996 é Engenheira de Projetos no Centro de Investigación Científica y Tecnológica del Ejército, Equador. Atualmente é Professora de Engenharia Eletrônica na Escuela Politécnica del Ejército.

José Carlos da Silva Lacava nasceu em Caçapava, SP, em 1951. Graduou-se em Engenharia Elétrica (opção Eletrônica) pela Faculdade de Engenharia de São José dos Campos em 1974. Obteve os títulos de Mestre em Engenharia Eletrônica (1979) e Doutor em Ciências (1985) pelo Instituto Tecnológico de Aeronáutica (ITA), São José dos Campos. É Professor Adjunto da Divisão de Engenharia Eletrônica e atual Chefe do Departamento de Microondas e Optoeletrônica do ITA. Em 1976 ingressou como Auxiliar de Ensino no ITA. Entre 1986 e 1987 foi Pesquisador Adjunto do Instituto de Atividades Espaciais desenvolvendo pesquisa em redes cilíndricas de antenas de microlinha para os canais de telemetria dos foguetes Sonda IV e VLS. De 1992 a 1993 foi Professor Associado Convidado da Universidade da Beira Interior, Covilhã. Portugal. Entre 1993 e 1994 foi Professor Assistente Doutor da Universidade Estadual Paulista "Júlio de Mesquita Filho" - Faculdade de Engenharia de Guaratinguetá. Teoria eletromagnética, dispositivos passivos em microondas e antenas são suas áreas de interesse. $\dot{E}$ membro da Sociedade Brasileira de Eletromagnetismo e da Sociedade Brasileira de Microondas e Optoeletrônica.

Lucio Cividanes nasceu em Nova Friburgo, RJ, em 1953. Graduou-se em Engenharia Elétrica (especialização em Telecomunicações) pela Universidade Federal Fluminense em 1974. 
Obteve o título de Mestre em Engenharia Eletrônica e Telecomunicações em 1978 pelo Instituto Nacional de Pesquisas Espaciais (INPE) e Doutor em Ciências pelo Instituto Tecnológico de Aeronáutica (ITA) em 1992. Atua como engenheiro da Divisão de Eletrônica Aeroespacial do INPE, trabalhando no desenvolvimento de antenas e circuitos de microondas para satélites. Tem experiência internacional na ESA (European Space Agency), estagiando no European Space and Tecnology Center (ESTEC) na Holanda, e trabalhando na firma SPAR no Canadá. Atuou também no Satélite Sino-Brasileiro de Recursos Naturais (CBERS), lançado em 1999, tendo participado na China de diversas atividades de projeto, fabricação e testes desse satélite. Suas principais áreas de interesse são antenas microstrip, redes planares e circuitos associados para aplicação espacial. É membro da Sociedade Brasileira de Microondas e Optoeletrônica. 